\title{
Early oncocytic adenocarcinoma of the transverse colon
}

A 69-year-old woman presented with abdominal pain. At colonoscopy, a depressed lesion with a central raised nodule, approximately $10 \mathrm{~mm}$ in size, was identified in the transverse colon. Under magnifying narrow-band imaging, the lesion revealed a type IIIB capillary pattern (capillary pattern classification [1]), and a type $\mathrm{V}_{\mathrm{I}}$ pit pattern (Kudo's classification [2]) was detected under magnifying chromoendoscopy using $0.05 \%$ crystal violet staining (๑ Fig. 1).

The endoscopic and clinical diagnosis of this lesion was submucosal colon cancer. Histologic biopsy evaluation demonstrated a moderately to poorly differentiated adenocarcinoma. For treatment the patient underwent laparoscopic colectomy. Histologic examination of the surgical specimen revealed characteristic tumor cells with eosinophilic cytoplasm arranged in a tubular pattern ( $\bullet$ Fig. 2).

The tumor cells were cuboid to columnar in shape, with a prominent round nucleolus within a round nucleus. The tumor cells invaded the submucosa without vascular invasion; the surgical margins were tumor-free. There was an adenoma component at the peripheral area of the tumor. A clear border was evident between the adenoma component and the unusual eosinophilic cells. There was no lymph node metastasis. Immunohistochemically, tumor cells revealed immunoreactivity for cytokeratin 20, carcinoembryonic antigen (CEA), and mitochondrial antigen, along with nuclear immunoreactivity for p53 in more than $50 \%$ of the neoplastic cells ( $\bullet$ Fig. 3).

Ki-67 stained positive in $10 \%$ of the neoplastic tumor cells. We diagnosed oncocytic adenocarcinoma. The patient was disease-free after 2 years.

Except in the thyroid and kidney, neoplasms composed of oncocytic cells are generally rare. The oncocytic variant of colorectal adenocarcinoma has been reported only twice $[3,4]$, and in both cases it was advanced. In those cases the tumor showed immunoreactivity for CDX2, CEA, cytokeratin 20, and a low Ki-67 labeling index, which accords with our case. Additional immunohistochemical analysis for chromogranin A, synaptophysin, and neuron-specific enolase suggested no endocrine differentiation. Oncocytic features
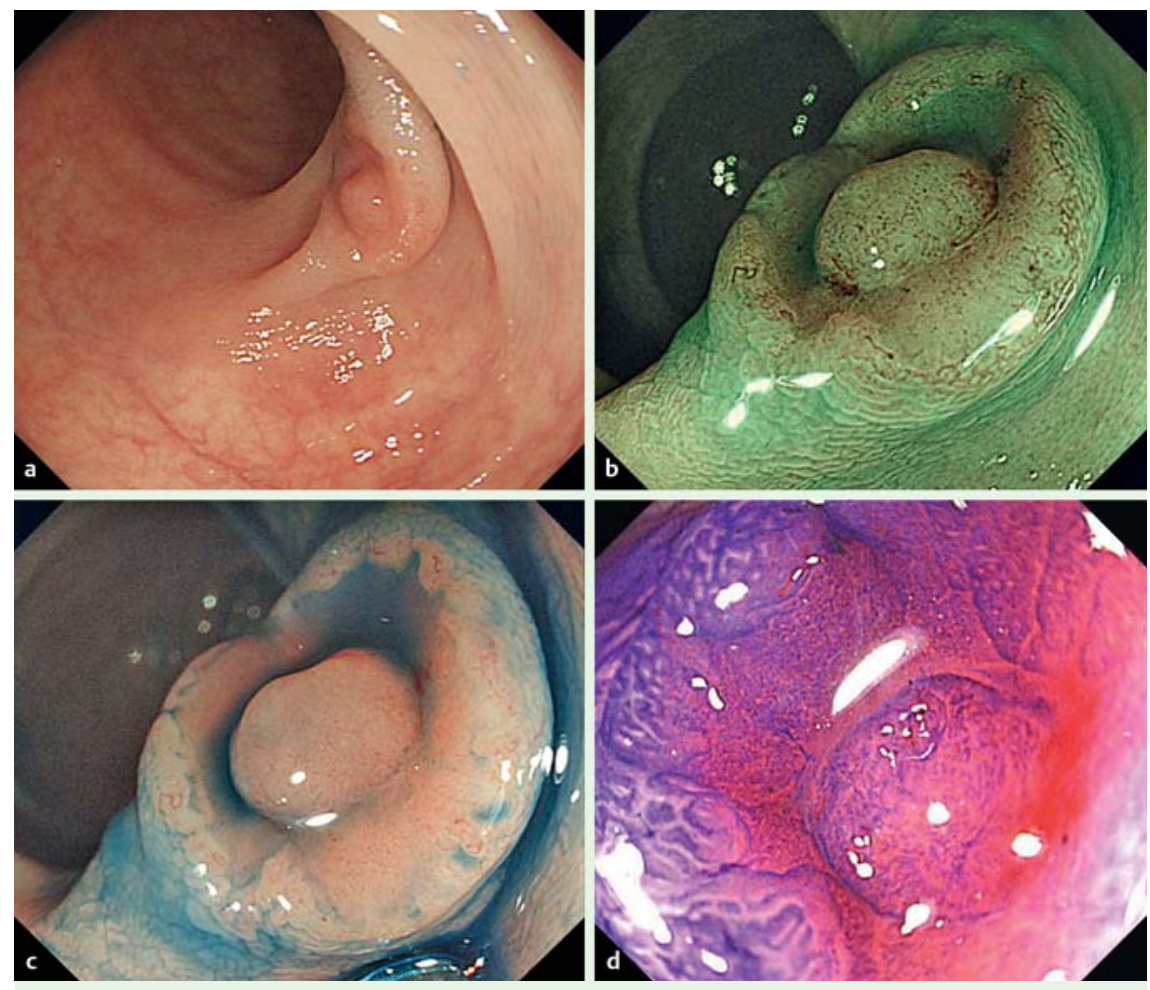

Fig. 1 Endoscopic view showed a depressed lesion in the transverse colon. Magnification with chromoendoscopy using $0.4 \%$ indigo carmine or $0.05 \%$ crystal violet staining revealed a type $V_{1}$ pit pattern in the depressed area. a Conventional view. b Narrow-band imaging view. c Chromoscopic view with $0.4 \%$ indigo carmine. d Magnifying view with $0.05 \%$ crystal violet staining.

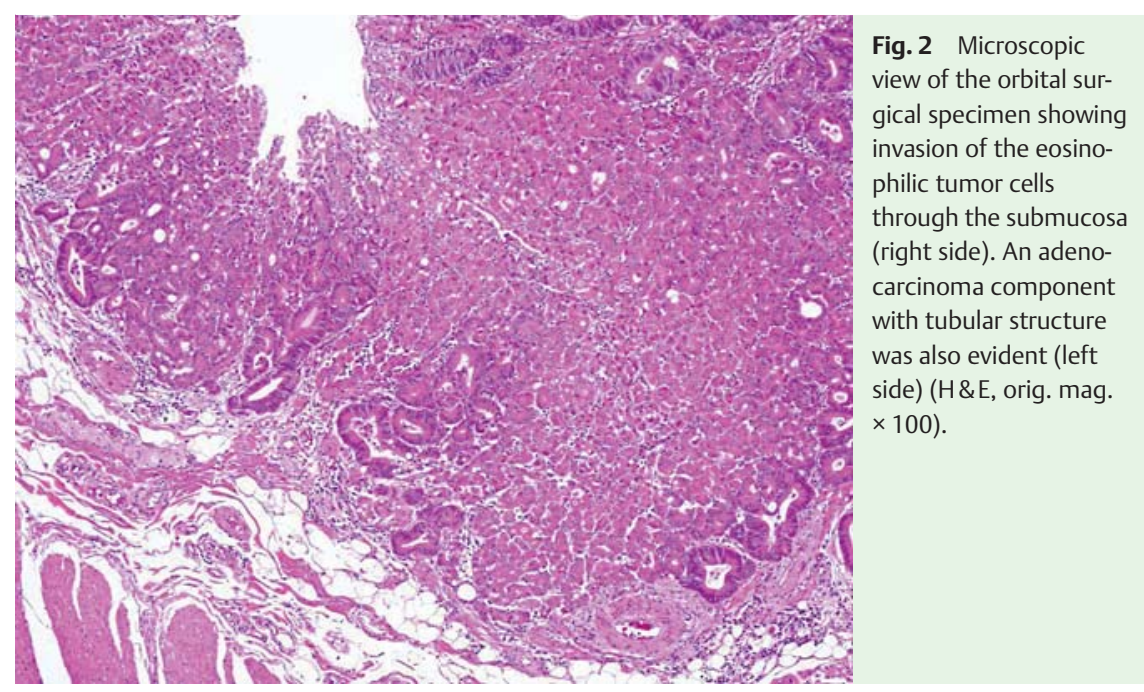

Fig. 2 Microscopic view of the orbital surgical specimen showing invasion of the eosinophilic tumor cells through the submucosa (right side). An adenocarcinoma component with tubular structure was also evident (left side) (H\&E, orig. mag.

were reported in a patient who had received preoperative chemoradiotherapy [5]. However, our patient did not receive preoperative chemoradiotherapy. Further, lack of nuclear pleomorphism and vesicular changes may be distinct from oncocytic features that develop after che- moradiotherapy. Our case, which shows identical histological and immunohistochemical features to previous cases, is the first case of early-stage oncocytic adenocarcinoma to be reported. This case shows that some early oncocytic adenocarcinomas may present as depressed lesions. 


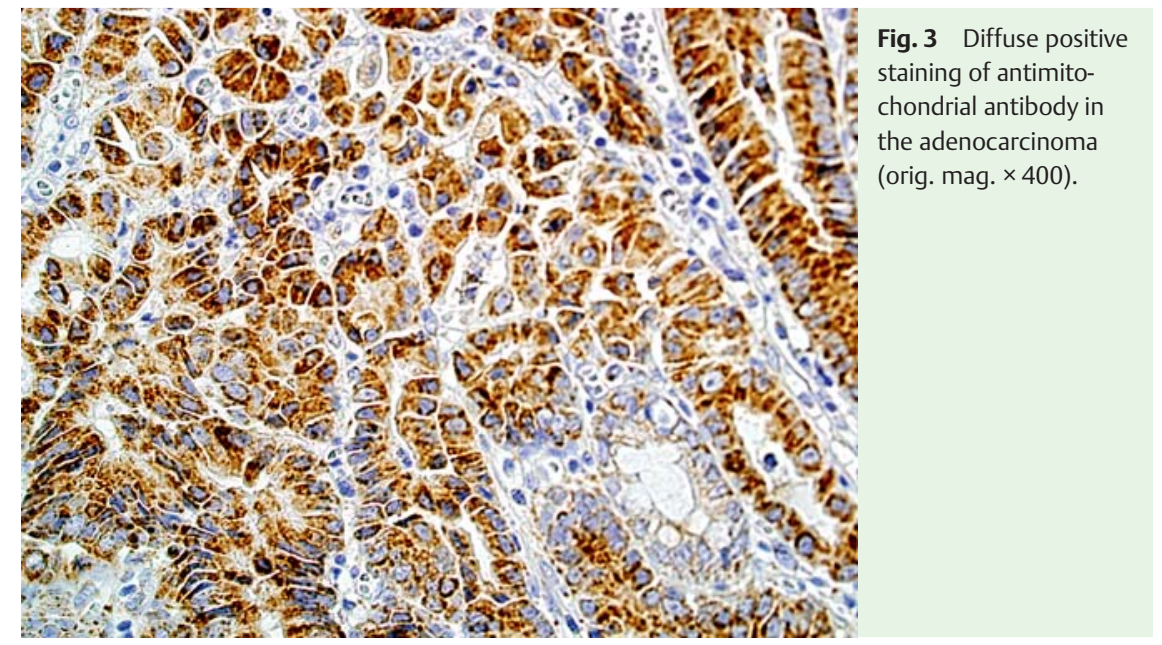

\section{Competing interests: None}

Endoscopy_UCTN_Code_CCL_1AD_2AC

D. Fukuda ${ }^{1}$, H. Ikematsu ${ }^{1}$, M. Kojima², Y. Oono ${ }^{1}$, T. Kojima ${ }^{1}$, K. Minashi ${ }^{1}$, T. Yano ${ }^{1}$, A. Ochiai ${ }^{2}$, K. Kaneko ${ }^{1}$

1 Division of Division of Digestive Endoscopy and Gastrointestinal Oncology, National Cancer Center Hospital East, Chiba, Japan

2 Pathology Division, National Cancer Center Research Institute East, Chiba, Japan

\section{References}

1 Ikematsu H, Matsuda T, Emura F et al. Efficacy of capillary pattern type IIIA/IIIB by magnifying narrow band imaging for estimating depth of invasion of early colorectal neoplasms. BMC Gastroenterol 2010; 10: 33

2 Kudo S, Rubio CA, Teixeira CR et al. Pit pattern in colorectal neoplasia: endoscopic magnifying view. Endoscopy 2001; 33: 367-373

3 Piscitelli D, Ingravallo G, Resta L et al. Oncocytic adenocarcinoma of the rectum with diffuse intra-luminal microcalcifications: the first reported case. Virchows Arch 2003; 443: 579-582

4 Piana S, Asioli S, Foroni M. Oncocytic adenocarcinoma of the rectum arising on a villous adenoma with oncocytic features. Virchows Arch 2006; 448: 228-231

5 Rouzbahman M, Serra S, Chetty R. Rectal adenocarcinoma with oncocytic features: possible relationship with preoperative chemoradiotherapy. J Clin Pathol 2006; 59: 1039 1043

\section{Bibliography}

DOI $10.1055 / \mathrm{s}-0030-1255658$

Endoscopy 2010; 42: E261 - E262

(c) Georg Thieme Verlag KG Stuttgart · New York . ISSN 0013-726X

\section{Corresponding author \\ H. Ikematsu, MD}

Division of Digestive Endoscopy

and Gastrointestinal Oncology

National Cancer Center Hospital East

Kashiwanoha 6-5-1

Kashiwa

Chiba 277-8577

Japan

Fax: +81-4-71346928

hikemats@east.ncc.go.jp 